\title{
EchoGéo
}

\section{La conquête du littoral « indien » d'Afrique du Sud}

Temporalités, acteurs et modes de valorisation

\section{Sylvain Guyot et Julien Dellier}

\section{(2) OpenEdition \\ Journals}

Édition électronique

URL : https://journals.openedition.org/echogeo/7803

DOI : 10.4000/echogeo.7803

ISSN : 1963-1197

Éditeur

Pôle de recherche pour l'organisation et la diffusion de l'information géographique (CNRS UMR 8586)

\section{Référence électronique}

Sylvain Guyot et Julien Dellier, «La conquête du littoral « indien » d'Afrique du Sud », EchoGéo [En

ligne], 7 | 2008, mis en ligne le 28 octobre 2008, consulté le 31 juillet 2021. URL : http://

journals.openedition.org/echogeo/7803; DOI : https://doi.org/10.4000/echogeo.7803

Ce document a été généré automatiquement le 31 juillet 2021.

EchoGéo est mis à disposition selon les termes de la licence Creative Commons Attribution - Pas d'Utilisation Commerciale - Pas de Modification 4.0 International (CC BY-NC-ND) 


\title{
La conquête du littoral « indien » d'Afrique du Sud
}

Temporalités, acteurs et modes de valorisation

\author{
Sylvain Guyot et Julien Dellier
}

\section{Introduction}

1 Le concept de littoral peut se résumer par une situation d'interface (terre-océan). C'est "le lieu des contacts et des échanges, et c'est à ce titre, l'une des zones les plus concernées par les processus contemporains de globalisation. La zone côtière est un espace où la compétition est si intense entre les hommes que le droit ne suffit plus à en gérer les conflits d'usage sans toujours chercher à concilier la protection, la mise en valeur et l'aménagement. $\aleph^{1}$ En Afrique du Sud, depuis le début de la colonisation au $\mathrm{XVII}^{\mathrm{e}}$ siècle, et de manière sans cesse renouvelée, le littoral est un espace de conquêtes " raciale $^{2} »$, culturelle, économique et conservationniste. Différentes temporalités (précolonial, colonial, apartheid, postapartheid), différents groupes en présence (Blancs, Noirs, tantôt dominants tantôt dominés) et différents types d'usages (portuaire, industriel, conservation, touristique, agricole...) font de ce littoral un véritable front pionnier (Beinart \& Hughes, 2007). On peut considérer la zone côtière sud-africaine, sur le temps long, comme étant « une forme spatiale témoignant d'un processus d'appropriation de nouveaux territoires, considérés comme un milieu vierge de toute trace de 'civilisation' occidentale $»^{3}$, caractéristique des pays neufs. L'ouverture sur l'océan reste d'ailleurs toujours associée symboliquement à un espace presque infini et peu maîtrisable, sujet à de multiples appropriations (Robbins, 2004) ${ }^{4}$. Cette recherche de l'infini océanique associée aux différentes phases de conquête passée du littoral sud-africain, associées à l'imposition de limites raciales (réserves indigènes, bantoustans, townships), de clôtures de réserves naturelles, de ports 
industriels construits ex-nihilo, nous conduit à utiliser les deux significations du terme de frontière pour caractériser cette zone côtière :

- la frontière ligne (border, boundary) exprimant tout ce travail de compartimentage et les discontinuités qui en résultent

- la frontière zone (frontier) symbolisant un contact flou, imprécis, mouvant entre des territoires littoraux en évolution et faisant l'objet de recompositions territoriales et de nouvelles conquêtes.

2 Dans cet article, nous considérons uniquement le littoral sud-africain correspondant aux rivages de l'Océan Indien, étirés sur $1850 \mathrm{~km}$ environ, localisés entre le Cap des Aiguilles (à environ 100km à l'est du Cap de Bonne Espérance) et Kosi Bay à la frontière avec le Mozambique. Par commodité de langage, nous le désignons comme étant le littoral « indien » d'Afrique du Sud, comme on parle du « littoral atlantique ».

3 Parler de conquête du littoral en Afrique du Sud suppose d'étudier la question sur le temps long afin de montrer en quoi le terme de conquête s'avère pertinent pour rendre compte des dynamiques territoriales littorales actuelles en Afrique du Sud. Les groupes non-blancs et en partie expulsés de la majorité des zones côtières au XIX ${ }^{\mathrm{e}}$ et $\mathrm{XX}^{\mathrm{e}}$ siècles prennent-ils leur «revanche » depuis la fin de l'apartheid, et sous quelle forme ? De même, les territoires littoraux "réservés " dans le passé pour ces populations sont soumis aujourd'hui à de nouvelles formes de conquête économique, potentiellement révélatrices du processus de connexion à la globalisation actuelle. Comment les analyser?

Pour tenter de répondre à ces questions, il nous a semblé important de contextualiser la conquête du littoral indien d'Afrique du Sud sur le temps long; en insistant sur l'importance des ressources environnementales côtières dont la volonté d'appropriation implique des rivalités d'occupation du littoral entre forces coloniales boers puis britanniques, avec quelques tentatives de littoralisation de la part des bantoustans ; pour expliciter enfin la réalité des nouvelles conquêtes littorales postapartheid associées à un désir de rééquilibrage territorial et de conquêtes électorales.

\section{La conquête du littoral « indien » par les Blancs : ressources, disputes et ripostes}

5 Selon une définition stricte, la zone côtière sud-africaine bordant l'Océan Indien s'étire donc sur $1850 \mathrm{~km}$. Le plus souvent, le Cap de Bonne Espérance est retenu comme limite occidentale. Ce littoral correspond à la terminaison australe du continent africain. De ce point de vue, il est associé à un ensemble de valeurs, stratégiques (lieu de passage et de contrôle maritime), symboliques (littoral ultime du bout du monde avant le continent Antarctique) et esthétiques. A ces valeurs sont associées des ressources environnementales

\section{Des ressources environnementales précieuses}

\section{Quelques données bioclimatiques}

Les qualités paysagères et les ressources environnementales de ce littoral sont remarquables malgré un nombre limité de ports naturels, dû à la prédominance de 
larges baies ouvertes. Un autre facteur limitant pour la navigation côtière s'explique par des conditions climatiques parfois extrêmes. En effet les perturbations tempérées $\mathrm{du}$ sud-ouest circulent en sens inverse du courant dominant ce qui a pour effet de produire de très grosses vagues saccadées. Le nombre d'épaves sur ces rivages peut l'attester. Ces difficultés en matière d'accessibilité renforcent le caractère "sauvage " de cette côte lié donc au relief et au climat.

7 En fonction du relief de l'arrière-pays (montagneux, collinéen ou plaine) les différentes sections côtières sont alternativement rocheuses ou sableuses (voir carte 1 les cartes couleur ont été envoyées en fichier séparé à cause de leur poids).

\section{Carte 1 - Le littoral « indien » d'Afrique du Sud - Côtes et ressources environnementales}

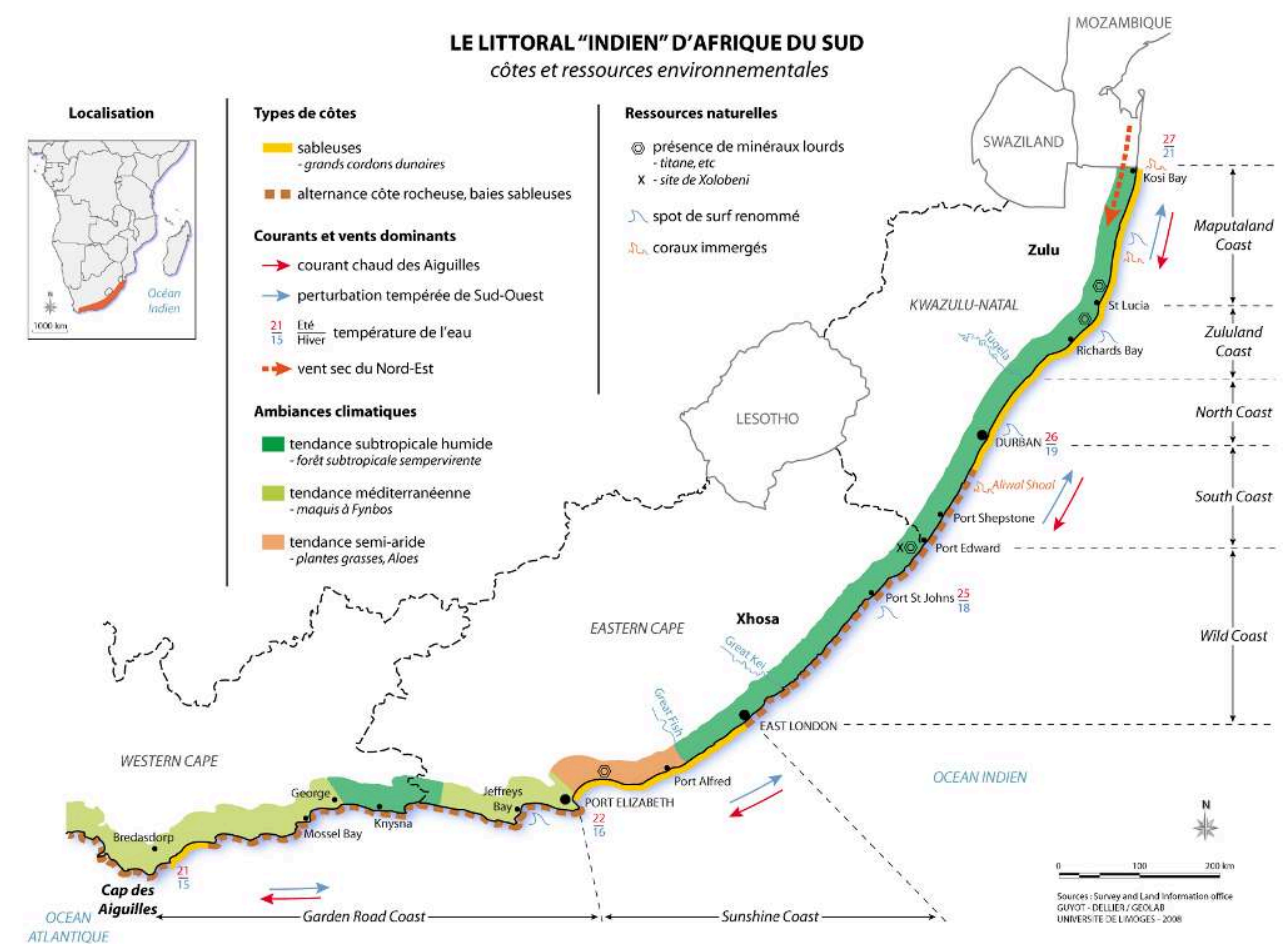

Pour impression

Sources : Survey and Land Information office ; Guyot-Dellier GEOLAB-UMR 6042, Université de Limoges.

8 On note toutefois une section continue de plusieurs centaines de kilomètres continus de plages au nord de Durban. Ce littoral est entièrement baigné par le courant chaud des Aiguilles qui «descend» du canal du Mozambique. De plus, la diversité ouest-est d'une palette climatique généralement tempérée permet à des écosystèmes littoraux méditerranéens (maquis à fynbos), tempérés chauds (prairie) et subtropicaux humides (forêt dunaire côtière, mangroves) de se développer avec une température de l'eau de mer toujours clémente (minimum occidental de $16^{\circ} \mathrm{C}$ et maximum oriental de $26^{\circ} \mathrm{C}$ ) et un ensoleillement globalement important. Le niveau élevé des précipitations sur ce littoral (plus d'un mètre d'eau par an), à l'exception de quelques sections à proximité du cap des Aiguilles et de la Sunshine Coast (à environ $500 \mathrm{~mm}$ ), garantit les récoltes. 


\section{Sols, sous-sols et océan}

9 La zone côtière, en arrière des cordons dunaires, dans les fonds de vallée et sur les plateaux côtiers dispose donc de sols fertiles pour l'agriculture et de gras pâturages pour l'élevage, sur la Wild Coast par exemple. Quand les sols ne sont pas aptes à l'agriculture, les sous-sols correspondant recèlent souvent de ressources minières remarquables. Ainsi, les cordons dunaires entre St Lucia et Richards Bay ou ceux de Xolobeni (photo 1), au sud de Port Edward, sont riches en minéraux lourds comme le titane ou l'ilménite.

Photographie 1 - La zone côtière de Xolobeni menacée par l'extraction du titane ? Wild Coast

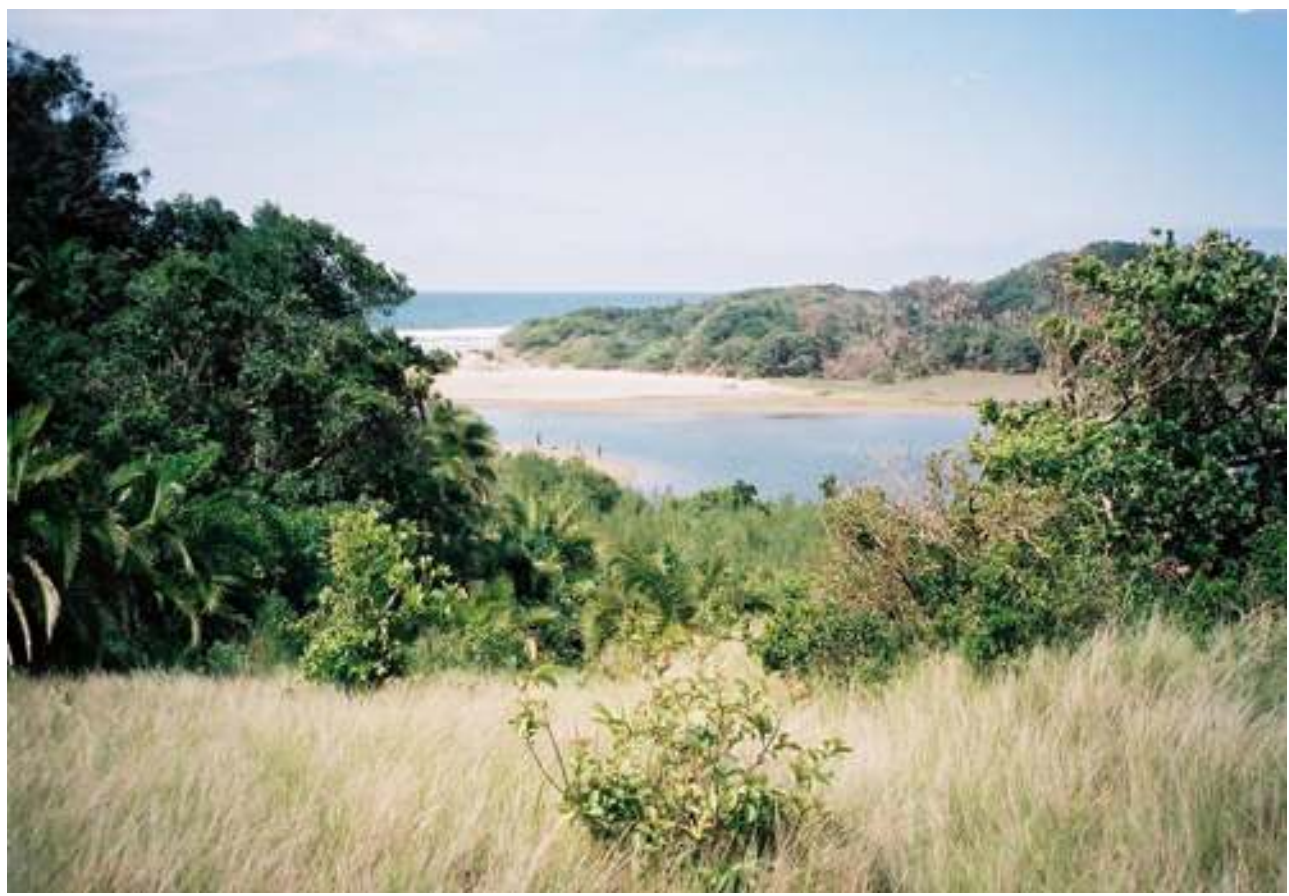

Cliché : Elisabeth Deliry-Antheaume.

La plate-forme océanique dispose de bonnes ressources halieutiques, en particulier lors du sardine run de juin-juillet, quand des milliers de poissons, requins, dauphins et baleines migrent vers les eaux du KwaZulu-Natal, certes à contre-courant des Aiguilles mais portés par la dérive littorale issue des perturbations tempérées du sud-ouest ${ }^{5}$. Certaines lagunes estuaires comme Kosi Bay ou St Lucia sont réputées pour être très poissonneuses. De plus, la côte à l'ouest de Port Elisabeth est réputée pour ses abalones (ormeau), espèce très recherchée de fruit de mer maintenant protégée par le gouvernement en raison de sa surexploitation. C'est aussi sur cette côte que l'on trouve les récifs coralliens immergés les plus austraux de la planète (Aliwal Shoal au large de la South Coast au KwaZulu-Natal), ainsi que des spots de surf mondialement réputés (Durban, Jeffreys Bay etc.).

11 Toutes ces ressources environnementales permettent de justifier l'intérêt de la conquête de ce littoral par différents groupes, à différentes périodes et pour différents types d'exploitation et/ou de valorisation. Les colons européens importent des modes et des idéologies d'aménagement littoral qui remettent en cause une configuration 
précoloniale structurée plutôt en retrait du littoral, à quelques exceptions notables près.

\section{Un « front pionnier littoral » contrôlé et disputé par les Blancs faisant suite à une configuration précoloniale assez peu littoralisée}

Durant la période précoloniale, la domination territoriale des espaces littoraux « indiens » d'Afrique du Sud est assurée par des groupes d'éleveurs de l'intérieur (Zulus au nord-est et Xhosas au sud-ouest de la rivière Umzimkulu). Les Zulus contrôlent le groupe littoral Tonga (implanté vers la frontière du Mozambique, voir Guyot, 2006) et les Xhosas s'allient au groupe Pondo (localisé entre les rivières Umzimkulu et Umzimvumbu), ceci leur permettant donc accès et exploitation des ressources marines (eau salée, pêche voir Mniki, 2007). D'autre part, la limite théorique entre populations bantoues et Khoisan serait localisée sur la rivière Fish, entre Port Elizabeth et East London et se stabiliserait au XVI ${ }^{\mathrm{e}}$ siècle, ce qui explique(rait) les moindres conflits et enjeux d'appropriation du littoral occidental à l'ouest de cette " frontière ». Toutefois, cette limite est bien sur discutée et discutable car elle a servit d'argument principal aux Blancs pour justifier leur colonisation d'une terre « vierge » à l'ouest de cette rivière, ce qui était sans tenir compte de la présence de populations Khoisan, chasseurs, cueilleurs mais aussi pêcheurs, répondant donc tout à fait à la définition de "peuple côtier ». N'ayant pas approfondi cette section occidentale du littoral « indien » dans nos recherches ${ }^{6}$ (Cape Agulhas et Garden Road Coast), nous insistons surtout dans cet article sur le littoral «indien» situé à l'est de Port Elizabeth (Sunshine, Wild, South, North, Zululand et Maputaland coasts).

C'est avec la colonisation que la conquête du littoral «indien » de l'Afrique du Sud devient un enjeu territorial fort. On pense immédiatement aux premières reconnaissances maritimes de B. Diaz (1487) et de V. de Gama (1497) à la fin du $\mathrm{XV}^{\mathrm{e}}$ siècle. Puis, les Boers ${ }^{7}$, s'implantent dans la province du Cap à la faveur d'une escale de la Compagnie hollandaise des Indes orientales ${ }^{8}$. Ils sont chassés au début du $\mathrm{XIX}^{\mathrm{e}}$ siècle par des Britanniques arrivés en force avec des idées libérales ${ }^{9}$. Les Boers vont alors tenter de reconquérir des nouveaux territoires à l'intérieur du pays mais aussi un accès à la mer, indispensable pour exporter certaines de leurs productions agricoles et pour assouvir leur passion pour la pêche. Ils quittent la région du Cap en caravanes via une route terrestre (le grand Trek), créent les colonies de l'Etat Libre d'Orange et du Transvaal, et tentent de former l'éphémère colonie littorale de Natalia, sur la côte du Natal, ayant du mal à résister aux forces britanniques malgré une victoire sur les Zulus. 
Carte 2 - Bilan des conquêtes littorales à l'aube du XXIe siècle - Prégnance des héritages territoriaux coloniaux et d'apartheid

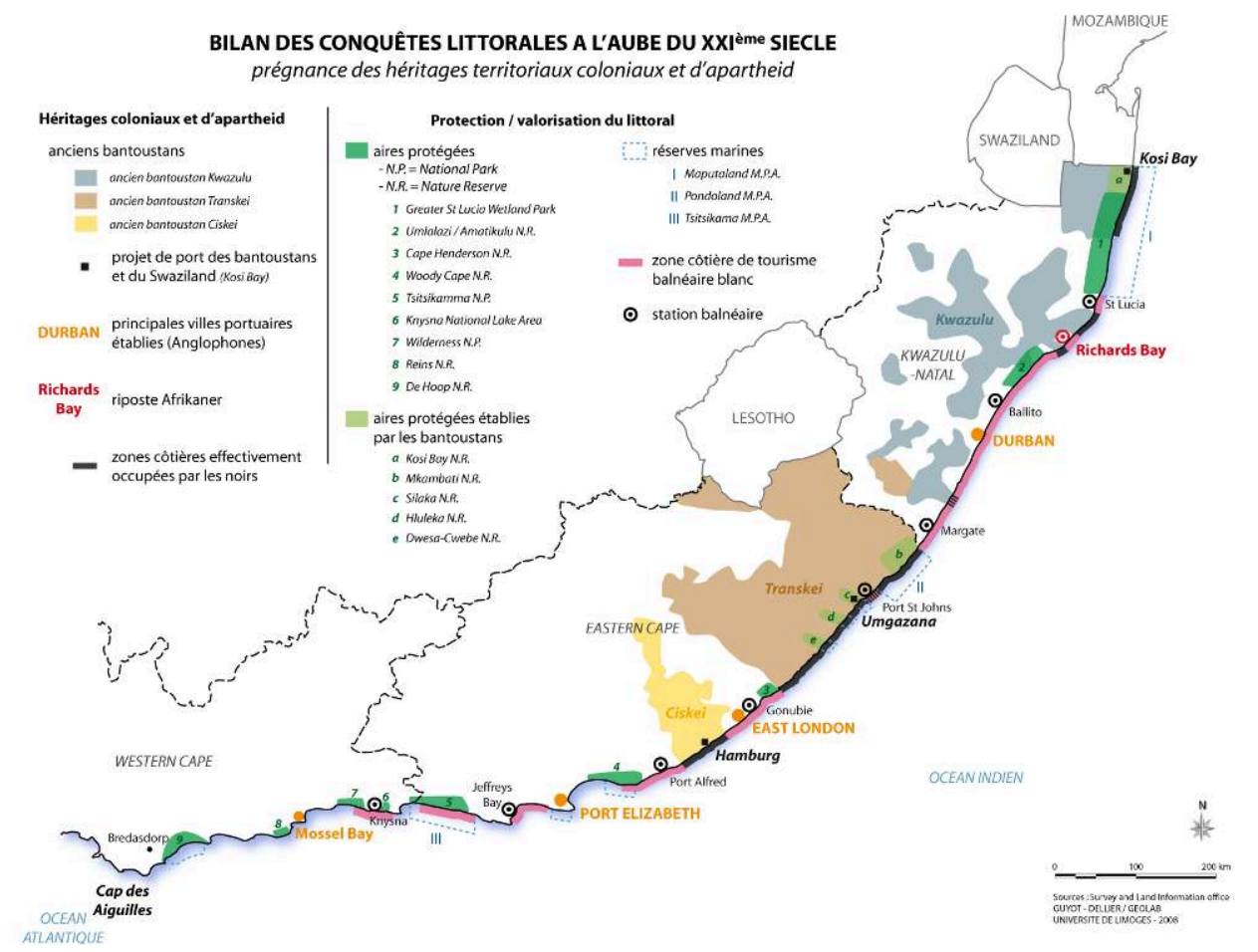

Pour impression

Sources : Survey and Land Information office ; Guyot-Dellier: GEOLAB-UMR 6042, Université de Limoges.

Les colons britanniques sont tentés, au XIX ${ }^{\mathrm{e}}$ siècle, de s'approprier l'ensemble des espaces littoraux sud-africains à partir du Cap, en créant sur le littoral « indien » plusieurs grands ports industriels servant d'exutoires pour les ressources minières intérieures: Port Elizabeth, East London, Durban (carte 2). Ces différents ports ${ }^{10}$ vont aussi servir de point de d'attache à la mise en valeur de l'arrière-pays et à la création des réserves africaines intérieures de petite taille (native reserves) dans la deuxième moitié $\mathrm{du} \mathrm{XIX}^{\mathrm{e}}$ siècle qui excluent presque totalement les populations africaines du littoral à l'exception notoire des côtes du Maputaland et du Transkei, même si Port St Johns (port éphémère de la Wild Coast au XIX ${ }^{\mathrm{e}}$ siècle) s'impose finalement comme une enclave blanche dans le Native Land Act de 1913. Cette appropriation littorale va se faire aussi sur le mode de la conservation de la «nature sauvage » (St Lucia Nature Reserve, déclarée réserve naturelle en 1897, une des plus anciennes d'Afrique du Sud) et du développement touristique balnéaire (George et sa route des jardins, Durban et ses North et South Coast, voir photo 2. On peut aussi mentionner l'arrivée massive de populations indiennes à la fin du XIX ${ }^{\mathrm{e}}$ siècle pour travailler sur les plantations sub-littorales de canne à sucre du Natal. Le littoral « indien » sud-africain s'indianise alors vraiment. Au-delà du jeu de mot, les indiens n'auront pas le droit de s'installer directement sur le littoral mais seulement en retrait (exemple de Tugela ou de Stanger). 


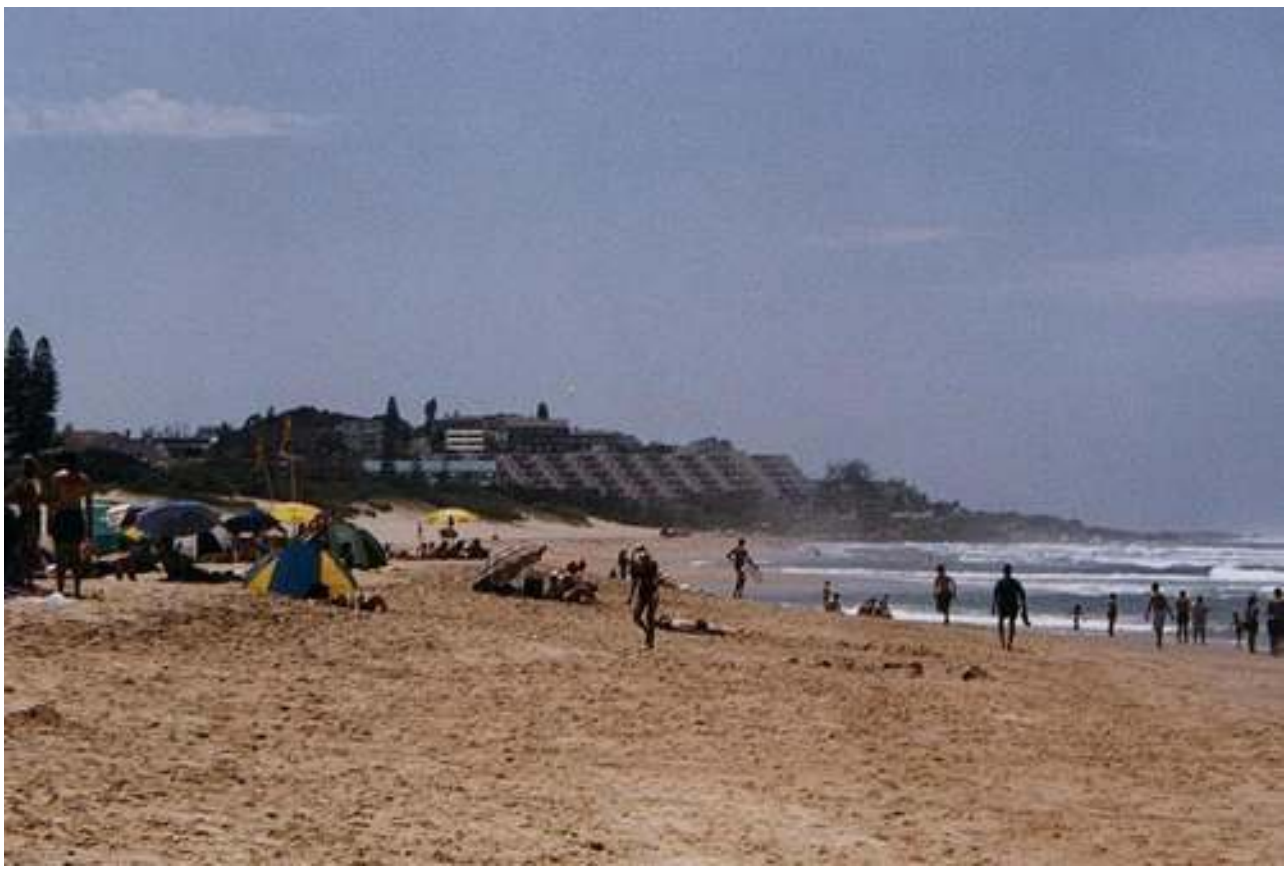

Cliché : auteur.

L'économie capitaliste mise en place en Afrique du Sud par les Britanniques contribue à réserver les espaces littoraux pour la résidence et les loisirs des classes blanches les plus aisées (sites urbains littoraux, aires protégées, plages) et pour assurer de bonnes connexions économiques entre ports sud-africains et le reste du monde. Les Boers, appelés Afrikaners depuis les années 1920-1930, connaissent un exode rural qui les conduit dans les grandes villes mais aussi sur les zones côtières à la recherche d'emplois liés au boom dans le secteur touristique comme c'est le cas sur le littoral entre Cape Town et Port Elisabeth ${ }^{11}$. Avant l'arrivée des nationalistes (surtout composés d'Afrikaners) au pouvoir en 1948, les Afrikaners ne contrôlent quasiment pas de linéaire littoral, entièrement géré par les intérêts anglophones (ex-britanniques).

\section{La riposte du gouvernement d'apartheid}

Durant l'apartheid, entre 1948 et 1994, le gouvernement nationaliste entérine la politique territoriale passée d'exclusion des populations africaines des zones économiquement stratégiques en Afrique du Sud. Mais il tente surtout de reconquérir "la mer promise ${ }^{12}$ ", abandonnée lors du grand Trek en 1837 (région du Cap) et définitivement perdue lors démantèlement de la colonie « Natalia » en 1843.

Cette reconquête de la «mer promise » s'illustre par plusieurs stratégies territoriales. Le contrôle des ports est confié à des cadres afrikaners avec la nationalisation de l'organisme de contrôle portuaire "Portnet ». D'ailleurs, l'accession massive à des fonctions administratives pour les Afrikaners dans les années 1950-1960 conduit à faire émerger une classe moyenne blanche numériquement importante. C'est ainsi qu'un tourisme littoral, plus tourné vers ces nouvelles catégories sociales émergentes, se met en place, avec par exemple, le développement massif de campings et de caravan parks ${ }^{13}$ en bord de mer, sur la Garden road coast, sur la South Coast. On assiste à une dynamique 
équivalente dans les aires protégées, où la pêche côtière redevient un véritable hobby pour les boers de sexe masculin. Jusqu'en 2002, ils ont d'ailleurs l'autorisation de circuler en $4 \mathrm{X} 4$ sur les plages pour aller pêcher. La station balnéaire de St Lucia s'impose dans les années 1980 comme un bastion afrikaner conservateur, très jaloux de son accès à l'estuaire et à la mer (Guyot, 2003). C'est aussi en termes de stratégies territoriale et politique de reconquête littorale qu'il faut comprendre le développement du port industriel de Richards Baai (Richards Bay, voir photo 3) ${ }^{14}$ en 1976, première Zone Industrialo Portuaire totalement contrôlée par les Afrikaners (Nicholas 1997, Guyot 2006).

Photographie 3 - Vue aérienne de Richards Bay

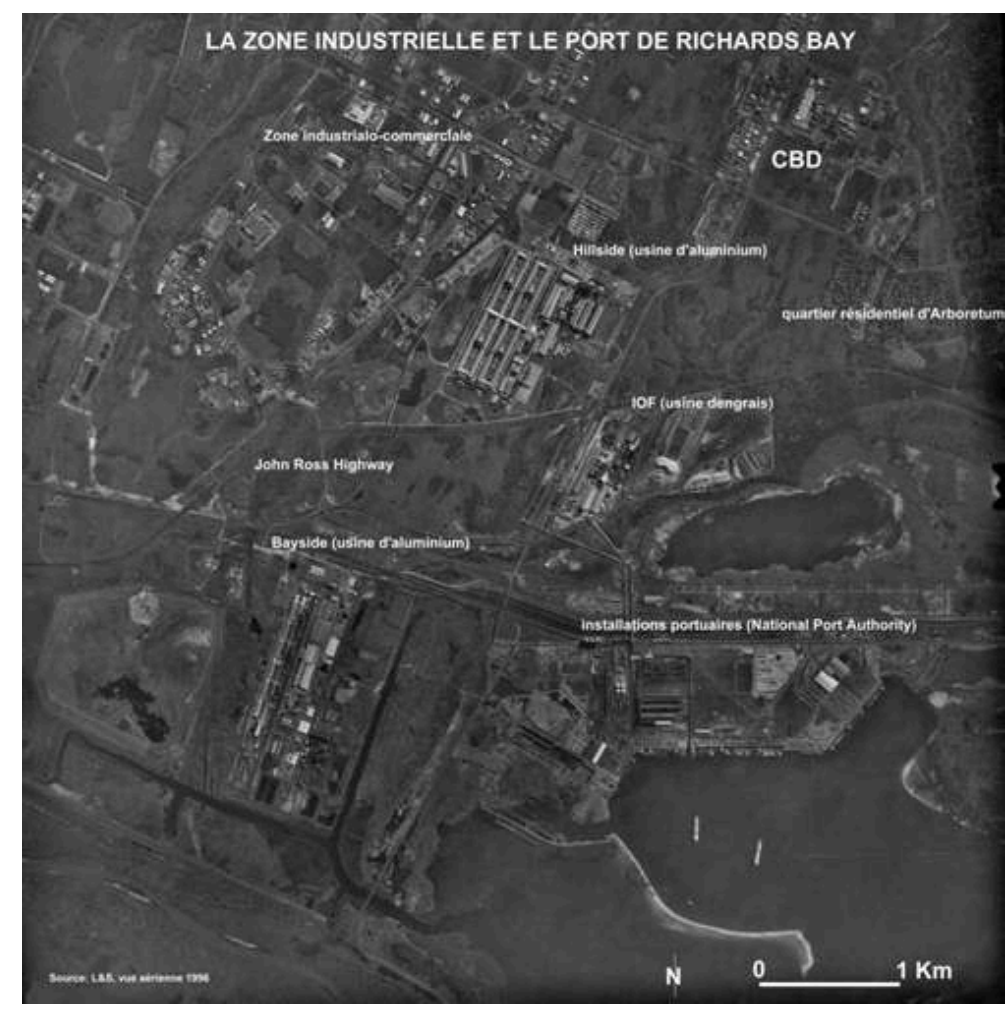

Cliché : Land \& Survey, 1996, retravaillé.

Dans le même temps, le gouvernement nationaliste poursuit les différentes politiques initiées par les Anglophones. C'est le cas pour la conservation de la nature avec l'existence d'un pacte d'union nationale entre conservationnistes anglophones et dirigeants politiques afrikaners lors de la création du «South African National Parks Board» en $1926^{15}$. La politique de création d'aires protégées se poursuit donc, en priorité sur les sections littorales les plus touchées par le tourisme balnéaire. Sur la Garden Road Coast, plusieurs parcs nationaux sont établis durant l'apartheid comme le Tsitsikamma National Park (photo 4) en 1964, le Knysna National Lake Area et le Wilderness National Park en 1985 (année de l'état d'urgence), qui, à eux deux, protègent plus de $140 \mathrm{~km}$ de côtes rocheuses et estuariennes entre George et Port Elizabeth. Le littoral s'impose donc totalement comme un territoire réservé aux Blancs. Pourtant, les quelques portions littorales africaines font entendre parler d'elles à partir des années 1976. 
Photographie 4 - Parc national de Tsitsikamma, Garden Road

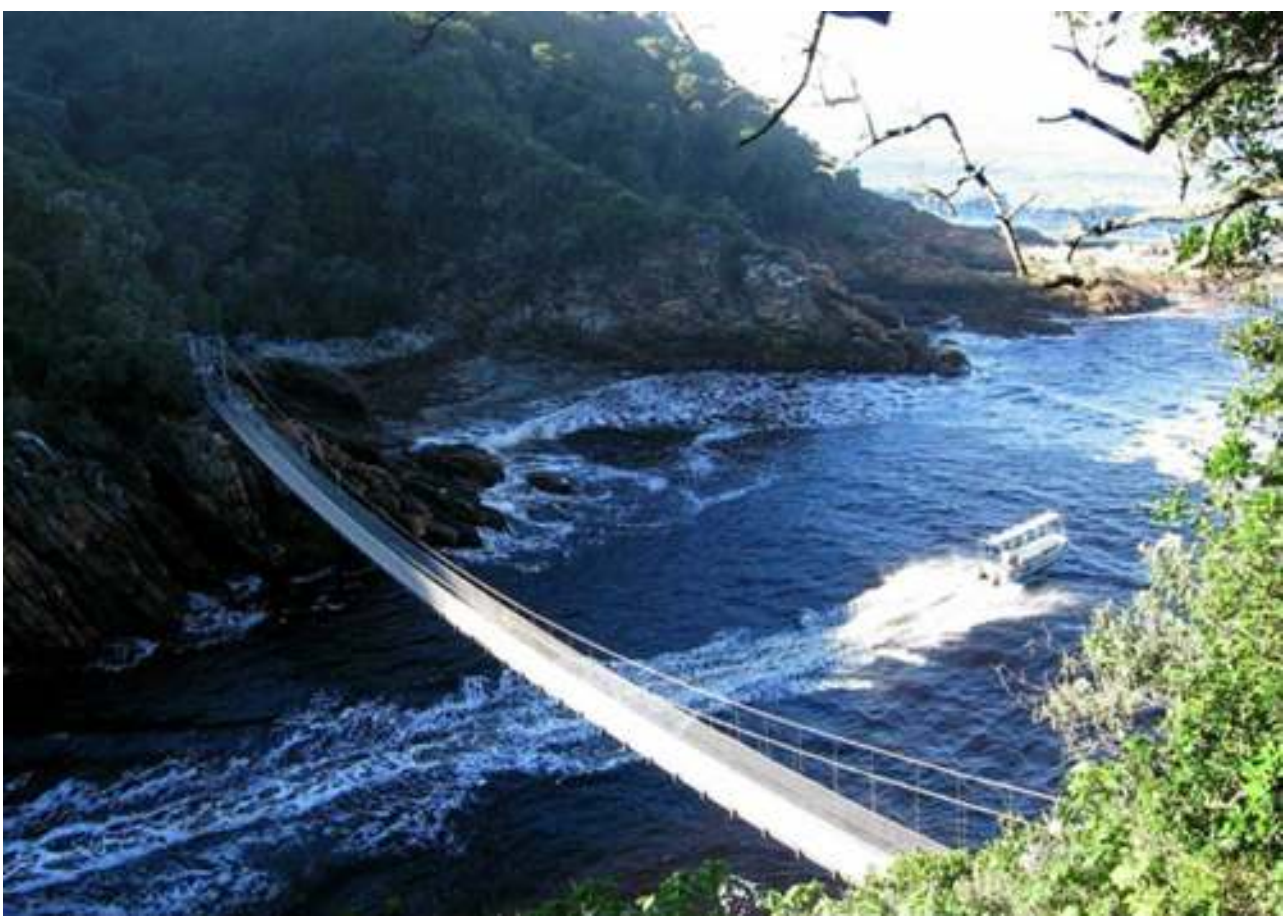

Cliché : auteur.

\section{Les « fantasmes littoraux » des bantoustans}

19 Au début des années 1970, le gouvernement nationaliste va édicter la politique du grand Apartheid, avec la création des bantoustans assignant chaque ethnie noire à résidence territoriale. Cette "escroquerie territoriale» va conduire les dirigeants « collaborateurs » africains à se poser des questions sur l'aménagement du littoral pour pouvoir peser économiquement. Ces Etats fantoches développent deux grands types de projets, portuaires et aires protégées, théoriquement de taille à peser sur l'aménagement des petites portions littorales africaines et sur le devenir économique des nouveaux Etats.

Le premier projet est la création de ports ${ }^{16}$ au Trankei et au Ciskei afin d'assurer à leur capitales respectives, Umtata et Bisho un débouché maritime. La localisation portuaire proposée pour le Transkei se localise à Mnagazana (estuaire d'un fleuve côtier) à $50 \mathrm{~km}$ au sud de Port St Johns. L'entrepreneur choisi par les nouvelles autorités du Transkei (contrôlées par le président Matanzima) pour réaliser ce port se nomme "Grands Travaux de Marseille ${ }^{17}$ " en relation avec la Société d'Etudes et d'Equipement d'Entreprises (Daily Dispatch, 15/10/1977). De même, au Ciskei, le président à vie Lennox Sebe, décide d'entreprendre un projet pharaonique de construction d'un grand port à l'estuaire de la Keiskamma (voir figure 1) au niveau du village de pêcheurs d'Hamburg. C'est une localité établie par des colons allemands à la fin du XIX ${ }^{\mathrm{e}}$ siècle puis abandonnée au Ciskei en 1981. Elle se trouve au sud d'East London ${ }^{18}$. Ces grands projets portuaires s'inscrivent évidemment dans la logique d'indépendance voulue par le grand Apartheid mais sont totalement irréalisables (voir surréalistes!) si on les ramène aux ressources propres et aux stratégies de développement économique de ces bantoustans. 
Figure 1 - Le projet de port à Hamburg, Ciskei

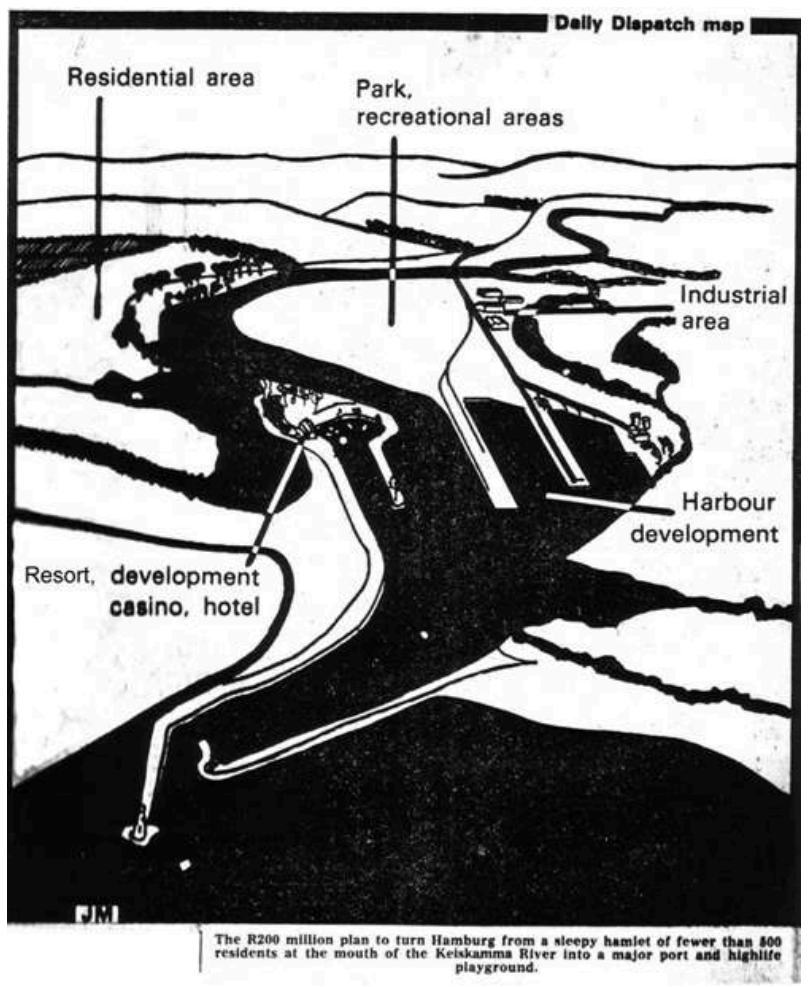

Source : presse locale d'époque.

Le second projet est l'établissement de nouvelles aires protégées littorales gérées par des organismes de conservation de la nature propres aux bantoustans. Dans le bantoustan autonome du KwaZulu, le KBNR (KwaZulu Bureau of Natural Resources) est une organisation de conservation favorable à la politique générale de l'Inkatha Freedom Party ${ }^{19}$ et de son leader M.G. Buthelezi. L'intérêt de l'IFP est de faire bénéficier les autorités tribales des revenus de la conservation pour en faire profiter les résidents. Le KBNR reconnait les chefs traditionnels comme les représentant légaux et directs des habitants. Les autorités tribales jugent positivement que les habitants soient dédommagés, et qu'ensuite ils disposent de $25 \%$ des revenus du parc à travers le système de " community levy " (Guyot \& Rey, 2003). En réalité, les gestionnaires et les scientifiques du KBNR sont des Blancs ayant une longue expérience de conservation, sensibles à la politique plutôt collaborationniste du bantoustan (Giraut, Guyot et Houssay, 2005). Ils utilisent cette structure pour établir de nouvelles réserves côtières au Maputaland : la Coastal Forest Reserve et la Kosi Bay Nature Reserve (photo 5). 
Photographie 5 - Estuaire de Kosi Bay, parcs de pêche traditionnels des populations Tonga, Maputaland Coast

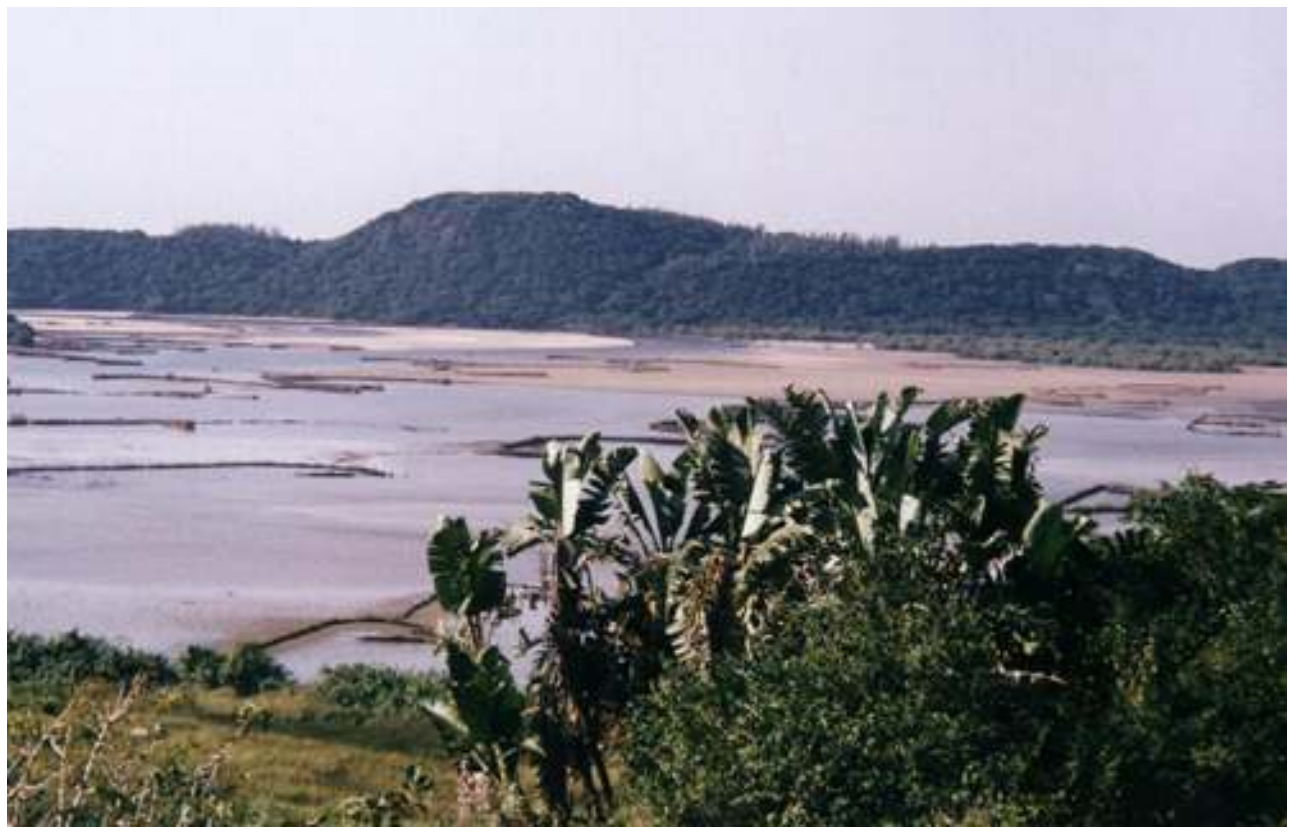

Cliché : auteur.

Malgré un système favorable aux populations locales prôné par le KBNR, ces dernières seront expulsées de leurs terres en 1989 pour laisser la place à la réserve naturelle de Kosi Bay, paradis pour pêcheurs blancs. De même, au Transkei, la réserve naturelle littorale de Mkambati est établie en 1977 en lieu et place d'une ancienne léproserie. Dans les années trois autres réserves naturelles littorales seront créées : Silaka près de Port St Johns, Hluleka plus au sud et Dwesa-Cwebe au sud de Coffee Bay. Cette dernière fait aussi l'objet d'expulsions de populations. La politique littorale des bantoustans s'inscrit donc totalement dans la continuité de la politique menée à Pretoria par le gouvernement nationaliste, l'appropriation du littoral se faisant, dans le meilleur des cas, au service d'une pseudo-élite politique noire et d'entrepreneurs blancs, mais surtout au service du développement séparé et de ses contradictions.

\section{Bilan de la conquête littorale à la fin de l'apartheid}

La fin de l'apartheid voit des compagnies minières, aux mains des hommes d'affaires blancs, surtout anglophones, prospecter les immenses richesses en minéraux lourds des dunes littorales (par exemple, société Richards Bay Minerals), se confrontant parfois à certaines oppositions véhémentes de leurs semblables (conflit contre l'extraction minière à St Lucia en 1992 mené avec succès par des écologistes anglophones). C'est aussi la période du développement de la pratique du surf grâce aux nombreuses vagues réputées du littoral sud-africain. Plusieurs spots majeurs apparaissent et drainent de nouveaux conquérants des vagues, tous blancs. Cette pratique sportive se développe à partir du spot pionnier de Durban avant de se propager sur l'ensemble du littoral «indien »: Cape St Francis, Jeffreys Bay jusqu'à Cape Town, et plus récemment vers Richards Bay et sur la côte du Maputaland (Mabibi). Les eaux chaudes baignées par le courant des Aiguilles sont évidemment privilégiées par les surfeurs. Là encore on note un décalage entre la dynamique interne de ces "près carrés littoraux de l'hédonisme 
blanc » et celle du reste du pays. Au milieu des années 1980, Nelson Mandela et d'autres hommes politiques noirs d'opposition sont emprisonnés sur l'île de Robben Island, ultime forme d'enfermement littoral, très symbolique de ces deux siècles de colonisation et d'apartheid. A la veille des premières élections libres en $1994^{20}$, on peut donc esquisser un bilan de la conquête du littoral par les Blancs.

Tableau 1 - Bilan des conquêtes littorales à l'aube du XXIe siècle : la force des héritages coloniaux et d'apartheid 監 minimisation des littoraux « noirs » et prégnance des aires protégées

\begin{tabular}{|c|c|c|c|}
\hline & & $\begin{array}{l}\text { Linéaire protégé } \\
\text { terrestre }\end{array}$ & $\begin{array}{l}\text { Linéaire protégé } \\
\text { maritime (réserves } \\
\text { marines) }\end{array}$ \\
\hline Linéaire littoral $\approx$ indien $\approx$ total & $1850 \mathrm{~km}(100 \%)$ & \multirow{2}{*}{$\begin{array}{c}650 \mathrm{~km} \\
\text { dont } 520 \mathrm{~km} \\
\text { correspondent } \\
\text { a une réserve marine }\end{array}$} & \multirow{2}{*}{$\begin{array}{c}650 \mathrm{~km} \\
\text { dant } 130 \mathrm{~km} \\
\text { ne correspondent pas } \\
\text { dune aire protegee } \\
\text { littorale terrestre }\end{array}$} \\
\hline Dont linéaire littoral protégé total & $\begin{array}{l}780 \mathrm{~km} \\
(42 \%)\end{array}$ & & \\
\hline Dont linéaire occupé par des Noirs & $505 \mathrm{~km}(27 \%)$ & \multicolumn{2}{|c|}{$\begin{array}{l}\text { Zone littorale protégee terrestre et/ou maritime } \\
235 \mathrm{~km} \text { (46\% du linéaire occupé par les Noirs) }\end{array}$} \\
\hline $\begin{array}{l}\text { Dont Zulus (rivages du Zululand et surtout du } \\
\text { Maputaland, ancien bantoustan KwaZZulu) }\end{array}$ & $180 \mathrm{~km} * *$ & \multicolumn{2}{|c|}{$115 \mathrm{~km}$ (64\% du linéaire Zulu) } \\
\hline $\begin{array}{l}\text { Dont Xhosa (groupe générique incluant les } \\
\text { Pondos, Thembus, Fingos etc: rivages des anciens } \\
\text { bantoustans du Ciskelet da Transkei) }\end{array}$ & $325 \mathrm{~km}^{* * *}$ & \multicolumn{2}{|c|}{$120 \mathrm{~km}$ ( $37 \%$ du linéaire Xhosa) } \\
\hline $\begin{array}{l}\text { Dont linéaire occupé par des Blancs } \\
\text { Difficile de faire précisement ía part des choses } \\
\text { entre peuplement afrikaner et peuplement } \\
\text { anglophone }\end{array}$ & $1345 \mathrm{~km}(73 \%)$ & \multicolumn{2}{|c|}{$545 \mathrm{~km}$ ( $40 \%$ du linéaire occupé par les Blancs) } \\
\hline \multicolumn{4}{|c|}{$\begin{array}{l}\text { - Les aires protégées sont soumises à des restrictions d'usage (développement touristique, prélèvement de fruits de } \\
\text { mer et pèche essentiellement). } \\
\text { ". Dont } 53 \mathrm{~km} \text { de linéaire cótier contrólé par Pentreprise Richards Bay Minerals qui extrait des minéraux lourds, Les } \\
\text { populations locales sont censées en bénéficier sous forme d'investissement social. } \\
\text { av } 20 \mathrm{~km} \text { de ce littoral, la zone de Xolobeni, pourrait servir de nouveau terrain d'exploitation minière à une entreprise } \\
\text { australienne. }\end{array}$} \\
\hline
\end{tabular}

Pour impression

Une marge d'erreur de +/- 5\% est à considérer sur les données présentées.

Source : auteur, à partir de calculs effectués sur des cartes appropriées (Atlas Sunbird 2007 \&

Recreational fishing information brochure 2005).

On évalue le pourcentage de littoral assigné aux différents groupes dans le tableau 1. A l'exclusion de toute forme de protection de la nature littorale (aire protégée terrestre et/ou réserve marine) seuls $270 \mathrm{~km}$ de linéaire littoral (soit seulement $14 \%$ ) peuvent être véritablement appropriés par les populations locales noires ${ }^{21}$. L'établissement d'aires protégées à proximité de zones rurales africaines induit de nombreuses contraintes pour les habitants en termes de prélèvement des ressources et de développement urbain touristique. En effet, la pêche, tout comme la collecte des fruits de mer, ou la cueillette des herbes médicinales traditionnelles sont soumises à des systèmes de permis et de quotas très contraignants. La fin de l'apartheid ne vient d'ailleurs qu'assouplir très faiblement cet ensemble de contraintes avec même, comme on le verra, une mise en concurrence des différents types de pêche (de loisir, commerciale et de subsistance) sur le même espace.

Il y a donc un fossé très important d'appropriation littorale entre les différents groupes de population sud-africains. Au total, $42 \%$ du littoral «indien » d'Afrique du Sud fait l'objet de mesures de conservation de la nature que ce soit sous la forme de parc national, de réserve naturelle et/ou de réserve marine ${ }^{22}$. C'est un chiffre très important qui consacre la zone côtière sud-africaine comme espace de tourisme et de loisirs de nature. C'est sur la foi de ces deux constats, inégalité blanc-noir d'accès et de résidence 
sur le littoral et prégnance de la zone côtière comme ressource environnementale et touristique, que sont basées certaines stratégies et politiques territoriales postapartheid.

\section{Les nouvelles conquêtes littorales postapartheid, entre stratégies politiques et globalisation}

26 La période postapartheid est la dernière phase de cette conquête du littoral ${ }^{23}$ où appropriations portuaires, industrielles, environnementales et touristiques continuent de s'affronter à travers l'affirmation conquérante de la nouvelle élite politique noire en relation parfois étroite avec les intérêts de la minorité blanche favorisée par la pleine connexion des espaces littoraux sud-africains avec la globalisation.

\section{Des stratégies politico-territoriales clairement littorales}

27 A la fin des années 1990, les SDI (Spatial Development Initiative) et les IDZ (Industrial Development Zones) sont deux éléments fondateurs de la politique postapartheid de renforcement, de redressement et de redistribution liée aux zones littorales en Afrique du Sud. Si les IDZ sont spécifiquement littorales car adossés à des ports ${ }^{24}$, les SDI (voir carte 3 comprennent tout de même 7 projets sur 10 impliquant des zones côtières (Lumbombo, Wild Coast etc.) ou ayant une finalité littorale (corridor de Maputo). Les SDI, Spatial Development Initiatives ${ }^{25}$ sont pilotées à partir de la Banque de Développement de l'Afrique Australe en collaboration avec le gouvernement national. Elles participent clairement à la revitalisation des zones littorales attractives mais marginalisées par le passé dans le cadre territorial des bantoustans (Lubombo SDI pour le KwaZulu et Wild Coast SDI pour le Transkei). Les SDI sont une stratégie pour renforcer des territoires moteurs socio-économiquement aux niveaux national et transnational (Guyot, 2006). Parfois, des objectifs purement politiques peuvent aussi se lire en filigrane de ces ambitions de développement. 
Carte3 - Dynamiques récentes du littoral « indien » d'Afrique du Sud

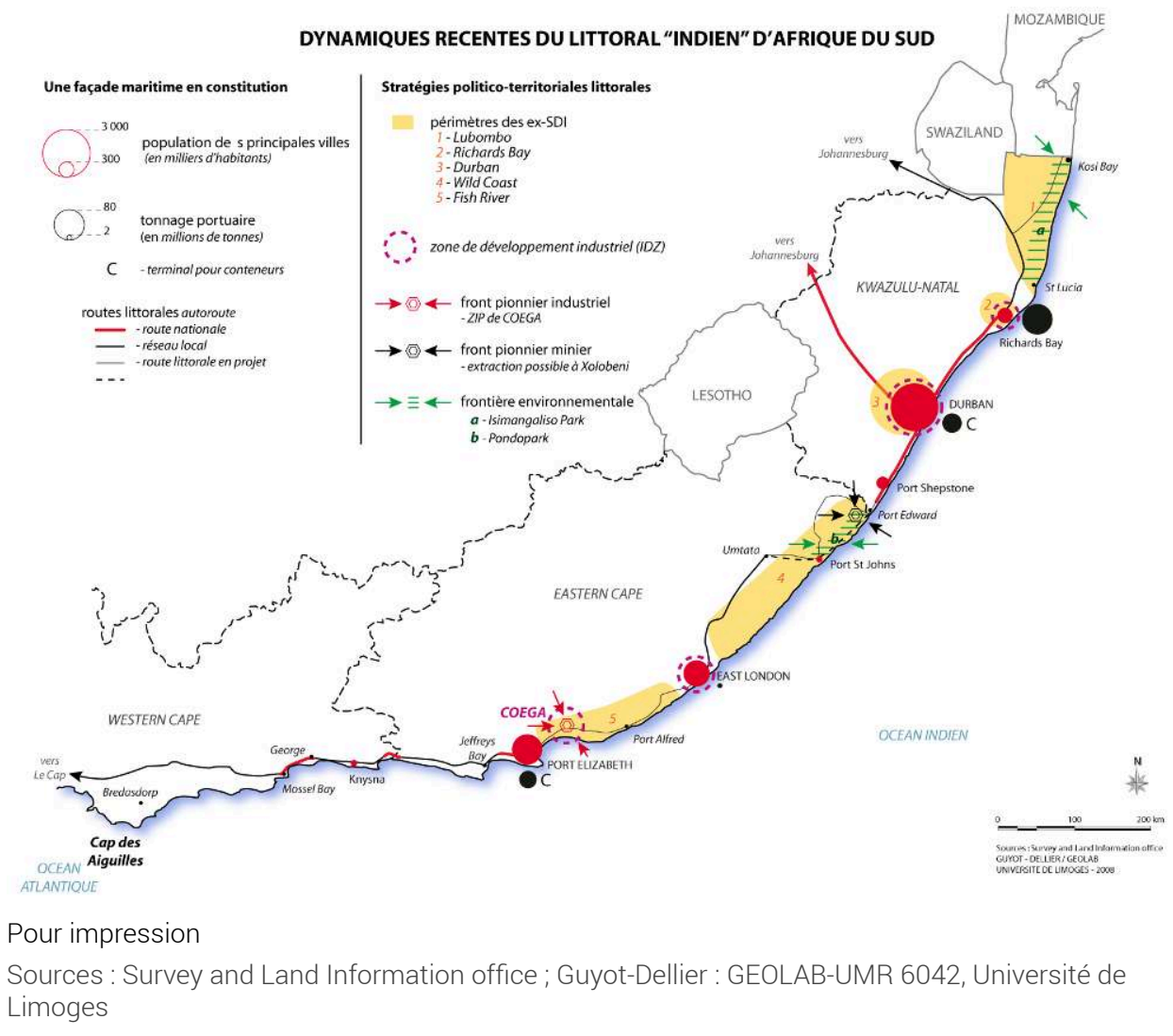

Le Greater St Lucia Wetland Park s'étend de St Lucia jusqu'à Kosi Bay à la frontière du Mozambique (voir carte 2). Le territoire du parc ainsi que sa zone périphérique sont chapeautés par le Lubombo SDI. Ce parc correspond à la réunion, sous le contrôle conjoint d'une Autorité Nationale et de l'organisme provincial de conservation (Ezemvelo KwaZulu-Natal Wildlife), d'une myriade de parcs et réserves naturelles aux statuts différents, certains rattachés à l'Afrique du Sud blanche et d'autres au bantoustan KwaZulu. L'unification de ce grand parc se décide suite aux fortes oppositions à l'extraction du titane dans les dunes de St Lucia au début des années 1990. La condition donnée par le gouvernement ANC en 1996 est de garantir le développement soutenu de l'écotourisme pour donner travail et services de bases aux populations riveraines fortement touchées par la pauvreté. Dans le même temps, le gouvernement ANC met en place une réforme territoriale au niveau local qui multiplie des institutions locales sans réels moyens financiers, comme pour les décrédibiliser au profit du niveau national. En créant des municipalités élues, il fait d'une pierre deux coups. Il donne aux chefs traditionnels des concurrents élus appartenant à la même mouvance, l'IFP, favorisant ainsi un conflit entre "anciens" et "modernes». Le gouvernement fait aussi la preuve que la structure municipale ne peut se passer de l'Etat central, faute de budgets suffisants, pour fournir les services de base à la population. Le gouvernement accélère donc la réalisation des aménagements routiers prévus dans le cadre du SDI pour montrer sa capacité de développement et d'intervention. D'un point de vue purement électoral les résultats de cette stratégie s'avèrent payants. Si on compare les résultats des élections locales de 2000, nationales de 2004 et locales de $2006^{26}$, l'ANC progresse dans toutes les municipalités incluses dans le Lubombo SDI. Par exemple au nord du parc, dans la municipalité de Manguzi, le score 
de l'ANC passe de $19,5 \%$ en 2000 à 45,4\% (score multiplié par 2,3) en 2006. Au sud du parc, dans la municipalité de Mtubatuba-St Lucia, le score de l'ANC passe de 18,9\% en 2000 à 36,8 \% en 2006 (score multiplié par 2). Dans chacune de ces municipalités, le score de l'IPF baisse d'autant. Le nouveau pouvoir national (ANC) prend le contrôle d'espaces littoraux largement fréquentés par les Blancs tout en essayant de capter l'électorat du principal parti noir d'opposition. Pour couronner le tout, en 2007, en phase avec la grande vague de changements toponymiques touchant l'Afrique du Sud (Giraut, Guyot et Houssay, 2008), le Greater St Lucia Wetland Park change de nom pour "Isimangaliso Park", devenant ainsi le premier grand parc littoral à l'identité africanisée.

Avec un décalage dans le temps d'une quinzaine d'années, on assiste à ce qui aurait pu être un remake de l'histoire de St Lucia, sur la Wild Coast. L'établissement d'un parc national (le PondoPark), mené tambour-battant en 2005 par des conservationnistes majoritairement blancs (réseau d'ONG 'Sustaining the Wild Coast') appuyés par le ministre blanc de l'environnement (J. Van Schalkwyk), se heurte vivement aux projets concurrents d'extraction minière dans la zone côtière de Xolobeni (photo 1) soutenus par les élus locaux de l'ANC (Guyot et Mniki, 2008; Guyot, Mniki et Dellier, 2007). Le gouvernement central semble également plus favorable à l'extraction minière qu'auparavant, celle-ci étant susceptible de créer plus emplois sur le court terme que l'écotourisme. Toutefois, la conquête minière ne tiendrait pas compte des droits fonciers des populations locales, qui, une fois de plus, seraient expulsées pour être relogées à l'intérieur des terres. Les mêmes méthodes passées semblent donc toujours de mise au XXIe siècle sur les littoraux sud-africains. De même, la construction du grand port industriel de Coega (figure 2) à $25 \mathrm{~km}$ à l'est de Port Elizabeth, faisant écho à l'épisode du développement de Richards Bay en 1976 par et pour les Afrikaners, représente la volonté expresse de développement industriel littoral du gouvernement dans une province symbolique, le Cap de l'Est, fief historique et électoral de l'ANC. Après de longues tergiversations, Coega attend toujours une réponse définitive du groupe ALCAN pour la construction d'une usine d'aluminium ${ }^{27}$ (figure 3). Si Jacob $Z u^{28}{ }^{28}$ est élu président de la République en 2008, peut-être que le centre de gravité du développement littoral sud-africain se redéployera vers le KwaZulu-Natal? La réalité est plutôt à la prégnance des forces exogènes sur le littoral « indien » d'Afrique du Sud quand littoralisation rime avec globalisation. 
Figure 2 - Affiche publicitaire vantant les mérites territoriaux et identitaires panafricains de COEGA

†wO thousami

YEARS AGO, A GREAT PORT

PLACED AFRICA

AT THE CEMTRE

OF WORLD TRADE.

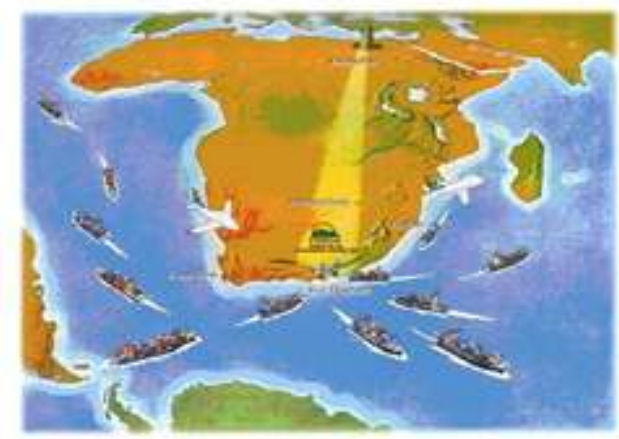

\section{HISTORY is ABOUT TO REPEAT ITSELF}

Source : www.coega.co.za

Figure 3 - Photographie aérienne et zonage spatial du développement de la ZIP de COEGA

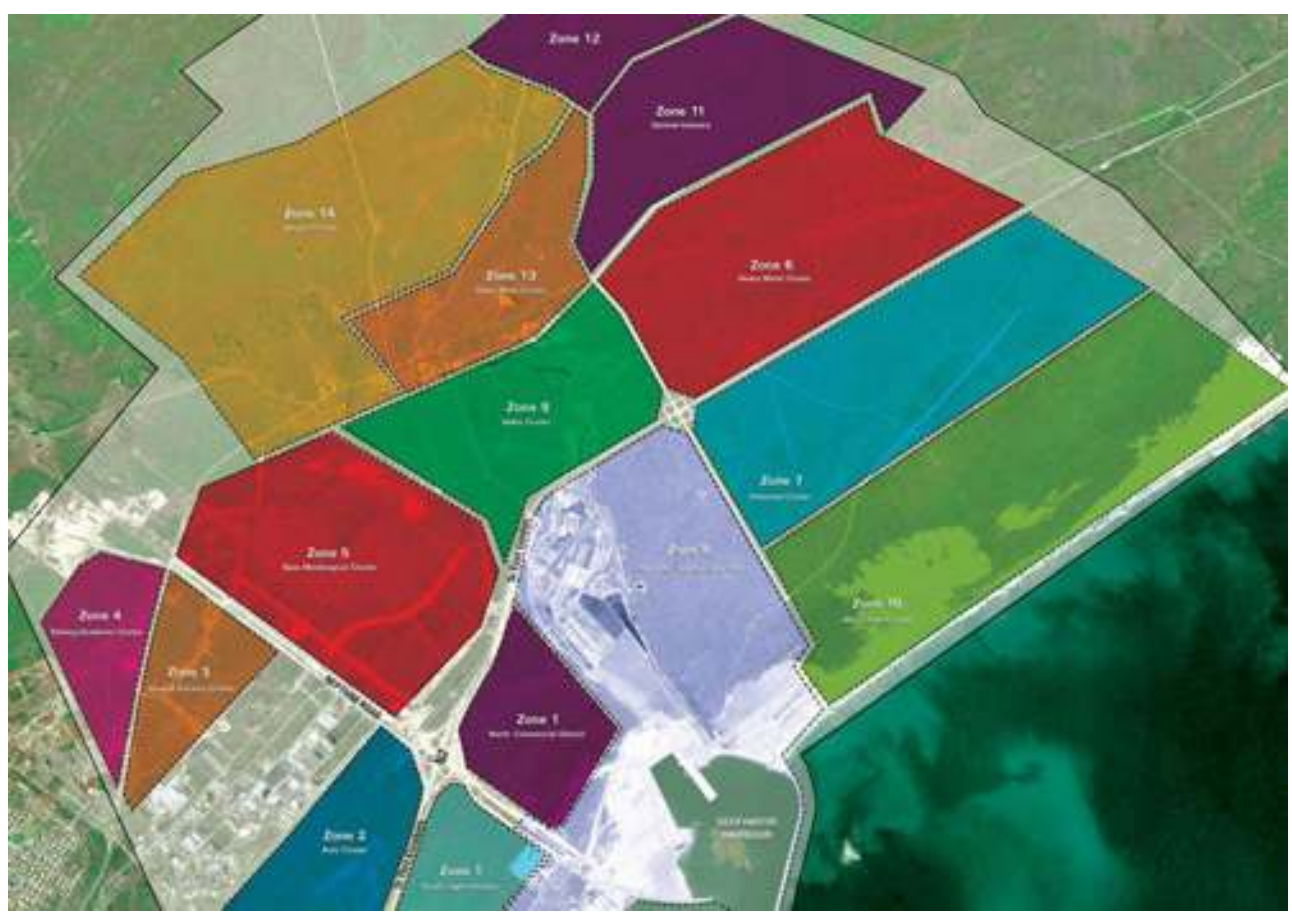

Source : www.coega.co.za 


\section{Entre poids des forces exogènes et action des masses !}

dernières convoitises en date exercées sur ce littoral sud-africain -émanent d'acteurs étrangers (ONG environnementalistes anglo-saxonnes, investisseurs immobiliers, touristes internationaux etc.) qui veulent aussi s'approprier leur part de cette bande côtière, exceptionnelle par ses ressources, ses paysages et ses populations. Le prix du foncier et des villas littorales (hors ex-bantoustan ${ }^{29}$ ) a considérablement augmenté sur les dix dernières années jusqu'à atteindre des niveaux occidentaux par endroits, comme sur le littoral autour de Durban, sur la Sunshine Coast (photo ${ }^{\circ} 6$ ) ou la Garden Road Coast.

Photographie 6 - Paysage littoral vers Port Alfred, Sunshine Coast

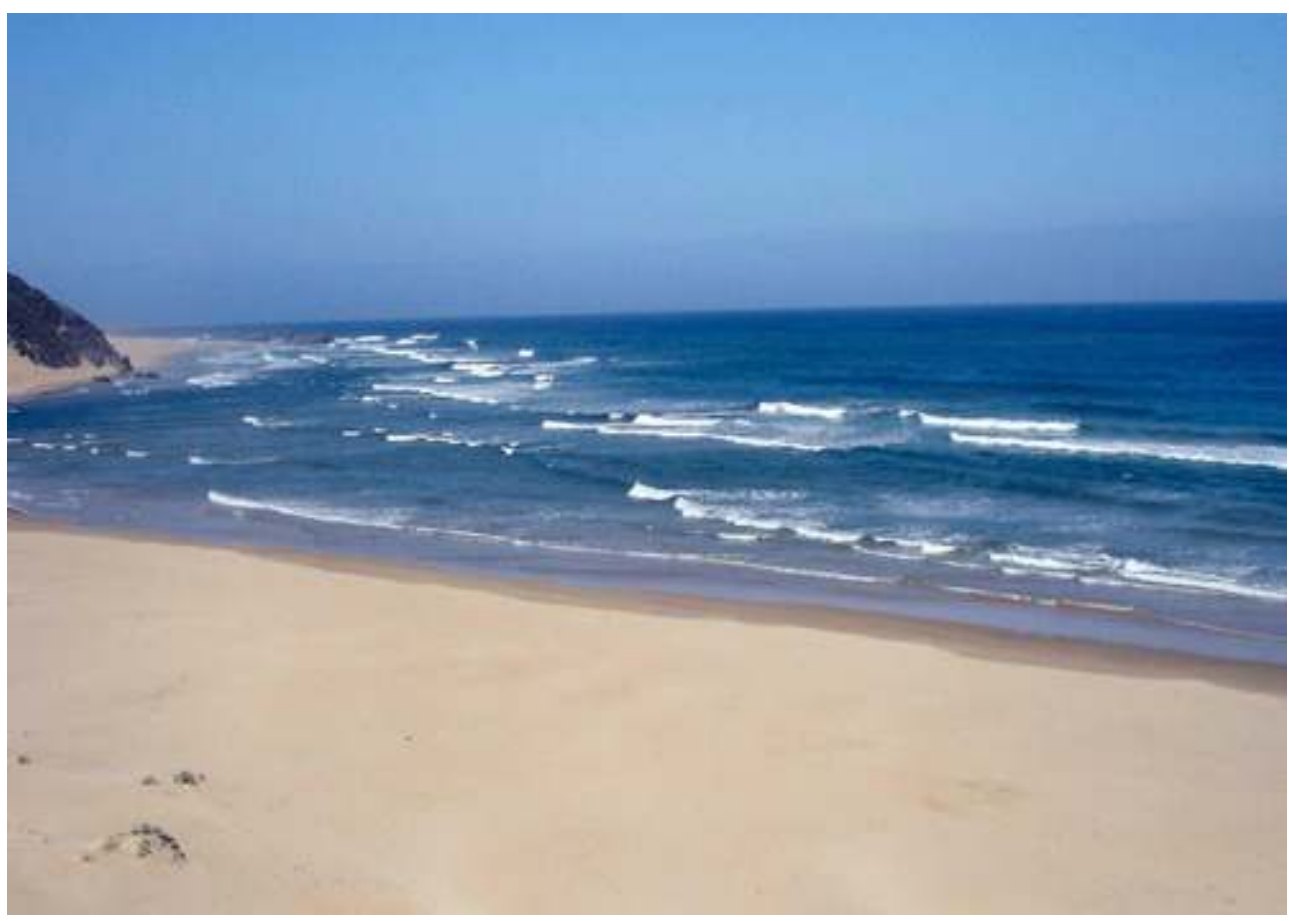

Cliché : auteur

31 Se pose aussi de manière cruciale la question de la privatisation des plages sudafricaines. La tendance passe en 15 ans d'une réservation raciale à une réservation sociale de l'espace balnéaire. Les blancs et les riches se baignent maintenant en priorité sur les plages que l'on trouve dans les aires protégées littorales où le droit d'entrée est payant et la sécurité garantie. $42 \%$ du littoral «indien» d'Afrique du Sud étant protégé, l'accessibilité en tous points de la zone côtière ne pose donc pas de problèmes pour ces clientèles triées sur le volet. A l'inverse, les plages publiques des villes, réservées pour les Blancs durant l'apartheid sont maintenant massivement appropriées par les Noirs des townships et des zones rurales avoisinantes. Au moment des fêtes, la densité de population atteint des records ce qui est un signe encourageant de réversibilité spatiale. Selon les endroits la gestion des flux est plus ou moins bien assurée $^{30}$ mais il est évident qu'en termes de représentations ce "mouvement d'appropriation inverse » ne fait pas plaisir à tout le monde ${ }^{31}$ (figure 4). Autre symbole ségrégatif qui éclate : la pratique du surf, elle aussi, se déracialise avec l'émergence de 
jeunes champions noirs même si le phénomène reste encore assez modeste, assez comparable avec celui de la pratique du rugby.

Figure 4 - La plage municipale de Richards Bay le 1 er janvier 2002, vue par la presse locale blanche

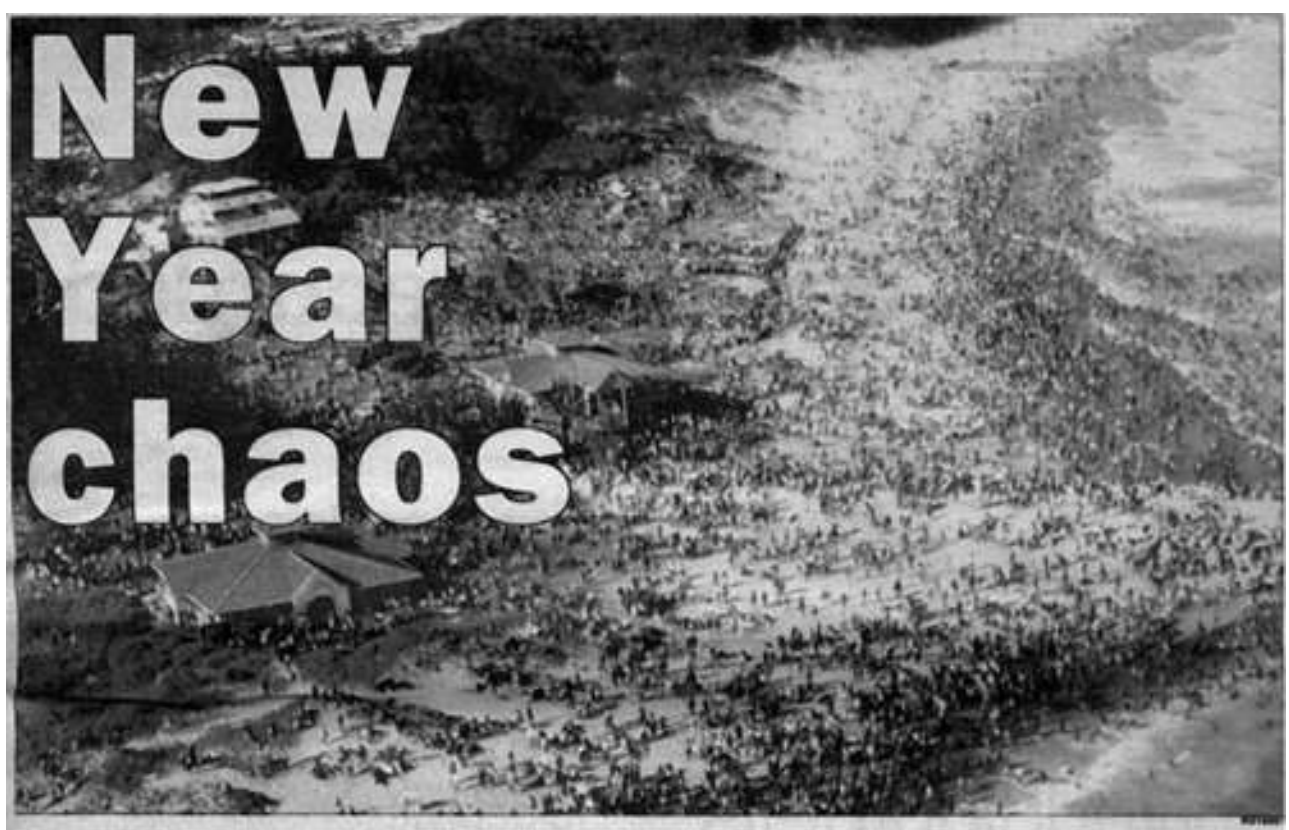

20 photographer Terbaa Pretortus took this photo of Alkantstrand on New Year's Day from the National Ports Authority helicopte

Source : Zululand Observer, presse locale.

Il n'y a véritablement que la pêche qui reste une activité partagée par tous les groupes de population sud-africains sur les mêmes espaces. Des blancs comme des indiens conduisent sur des centaines de kilomètres dans leurs bakkies ${ }^{32}$, équipés de congélateurs, pour aller pêcher sur la Maputaland Coast ou la Wild Coast. En raison de l'interdiction en 2002 des $4 \times 4$ sur les plages, ils sont contraints d'aller moins loin avec leurs véhicules et donc se postent à proximité des accès routiers, stations balnéaires ou hébergements d'aires protégées. De ce fait, ils rentrent en concurrence sur la ressource halieutique avec les pêcheurs commerciaux et de subsistance noirs. De manière assez injuste, l'ensemble de ces pêcheurs est soumis aux même réglementations et quotas de pêche. La condition de survie alimentaire par la pêche n'est pas reconnue par les autorités et oblige certains habitants à pêcher la nuit "au lamparo " (Mniki, 2007). L'augmentation des flux touristiques nationaux et internationaux sur les littoraux implique aussi un renforcement de la demande en poissons et autres crustacés ce qui, à terme, met en péril la ressource. L'aquaculture se développe, non sans interrogations et conflits sur ses modes de développement et de gestion ${ }^{33}$.

33 La littoralisation des populations africaines, qu'on espérerait synonyme d'un meilleur accès au littoral et de partage des bénéfices réalisés, est encore soumise à d'énormes contraintes et ce malgré la démocratisation du pays et la création de nouvelles municipalités « intégrées » regroupant zones côtières et arrière-pays. 


\section{Conclusion}

La conquête du littoral «indien » d'Afrique du Sud est un processus qui s'accomplit et se renouvelle depuis les débuts de la colonisation du pays jusqu'à nos jours (voir tableau 2). Chaque groupe au pouvoir, britanniques puis anglophones du parti uni, afrikaners du parti national puis africains de l'ANC, cherche à s'approprier le littoral et à imposer sa marque territoriale. La difficulté pour les gouvernants d'aujourd'hui est de gérer l'héritage de la période coloniale et d'apartheid avec seulement $27 \%$ du littoral « indien » effectivement "réservé » aux populations noires, sans tenir compte des contraintes liées aux aires protégées. Ces zones côtières sont marquées par un sousdéveloppement notoire en raison de leur ex-statut de réserve indigène et de bantoustan.

Tableau 2 - Temporalités, acteurs et valorisation du littoral « indien » d'Afrique du Sud

\begin{tabular}{|c|c|c|c|c|c|c|}
\hline Valorisation & Portuaire & Industrielle & Agricole & $\begin{array}{l}\text { Conservation de la } \\
\text { nature }\end{array}$ & $\begin{array}{l}\text { Tourisme } \\
\text { balnéaire }\end{array}$ & $\begin{array}{l}\text { «front pionnier } \\
\text { littoral » } \\
\text { principalement } \\
\text { disputé }\end{array}$ \\
\hline $\begin{array}{l}1652 \\
\text { Coloniale } \\
\text { (boer) }\end{array}$ & \multicolumn{2}{|c|}{$\begin{array}{l}\text { Le Cap } \\
\text { Escale, exutoire agricole, } \\
\text { coopératives viticoles }\end{array}$} & $\begin{array}{l}\text { Agriculture } \\
\text { plutôt } \\
\text { sublittorale, } \\
\text { élevage } \\
\text { Sunshine } \\
\text { Coast } \\
\end{array}$ & & & \multirow{2}{*}{$\begin{array}{l}\text { Côte du Natal } \\
\text { entre Boers, } \\
\text { Britanniques et } \\
\text { Zulus } \\
\text { Port St Johns, } \\
\text { enclave littorale } \\
\text { britannique sur la } \\
\text { côte du Transkei }\end{array}$} \\
\hline $\begin{array}{l}\text { Début XIX } \\
\text { Coloniale } \\
\text { (britannique et } \\
\text { anglophone) }\end{array}$ & \multicolumn{2}{|c|}{$\begin{array}{l}\text { Le Cap, Port Elizabeth, East } \\
\text { London, Durban }\end{array}$} & $\begin{array}{l}\text { Canne à } \\
\text { sucre littorale } \\
\text { et fruits } \\
\text { tropicaux } \\
\text { (Natal) }\end{array}$ & $\begin{array}{l}\text { St Lucia Nature } \\
\text { Reserve } 1897\end{array}$ & $\begin{array}{l}\text { Durban, } \\
\text { North Coast, } \\
\text { South Coast, } \\
\text { Garden } \\
\text { Road }\end{array}$ & \\
\hline $\begin{array}{l}1948 \\
\text { Apartheid }\end{array}$ & \multicolumn{2}{|c|}{$\begin{array}{l}\text { Richards Bay, Saldanha Bay, } \\
\text { Mossel Bay (pêche) } \\
\text { Projets des bantoustans, } \\
\text { Projet du Swaziland (Kosi } \\
\text { Bay) }\end{array}$} & $\begin{array}{l}\text { Périmètres } \\
\text { sublittoraux } \\
\text { (Maputaland } \\
\text { et Transkei) }\end{array}$ & $\begin{array}{l}\text { Réserves naturelles } \\
\text { des bantoustans } \\
\text { (KwaZulu, Transkei) }\end{array}$ & $\begin{array}{l}\text { St Lucia, } \\
\text { Sodwana, } \\
\text { South Coast } \\
\text { (Margate), } \\
\text { Sunshine } \\
\text { Coast } \\
\end{array}$ & $\begin{array}{l}\text { Zululand et } \\
\text { Maputaland } \\
\text { (bantoustan } \\
\text { KwaZulu) }\end{array}$ \\
\hline $\begin{array}{l}1994 \\
\text { Post-apartheid }\end{array}$ & IDZ, Coega & & Biocarburants & $\begin{array}{l}\text { Pondoland National } \\
\text { Park, extension } \\
\text { littorale d'Addo } \\
\text { Elephant Park, } \\
\text { changement de nom } \\
\text { du Greater St Lucia } \\
\text { Wetland Park. }\end{array}$ & $\begin{array}{l}\text { Ecotourisme } \\
\text { balnéaire } \\
\text { relié aux } \\
\text { aires } \\
\text { protégées } \\
\text { (Wild Coast, } \\
\text { Maputaland) }\end{array}$ & $\begin{array}{l}\text { Wild Coast } \\
\text { (ancien } \\
\text { bantoustan du } \\
\text { Transkei) }\end{array}$ \\
\hline
\end{tabular}

Pour impression

Cette nouvelle conquête d'un littoral parfois « oubliés " ", comparable au développement d'un nouveau front pionnier, se heurte aux modes de développement choisis, parfois environnementalement lourds ou socialement ségrégatifs (extraction minière, industrie lourde, aires protégées, tourisme balnéaire) et à un contexte plutôt agressif de globalisation marqué par une prégnance importance de forces exogènes. Les erreurs du passé en matière ségrégative ou environnementale (destruction versus conservation extrémiste) ont tendance à se répéter, sur fond de calculs électoralistes ou d'évolution des droits fonciers encore incertaine. 


\section{BIBLIOGRAPHIE}

Beinart W. et Hughes L., 2007, Environment and Empire, Oxford history of the British Empire, Companion Series, Oxford, Oxford University Press, 395 p.

Giraut F., Guyot S., Houssay-Holzschuch M., 2005, « La nature, les territoires et le politique en Afrique du Sud », Annales HSS, 60 année, n 4 , p.695-717.

Giraut F., Guyot S. et Houssay-Holzschuch M., 2008, « Enjeux de mots, les changements toponymiques sud-africains ", Espace Géographique, 2-2008, à paraître.

Guyot S., 2002, "Spatial competition and the new governance framework in Mabibi (Maputaland): implications for development”, Geographical Journal, vol. 168, n¹, p. 18-32, Carfax publishers.

Guyot S., 2003, « Les conflits environnementaux à St Lucia (Afrique du Sud), entre protection et exclusion », Annales de Géographie, n634, pp 608-627, Armand Colin.

Guyot S., 2004, « Derrière le masque de l'écotourisme, le politique : conservation et discrimination territoriale en Afrique du Sud », Revue Tiers-Monde, t. XLV, n¹78, p 341-363.

Guyot S., 2006, Rivages Zoulous: l'environnement au service du politique en Afrique du Sud, Paris, Karthala et IRD, 256 p.

Guyot S., Mniki L., Dellier J., 2007, “The Wild Coast: last frontier for South African nature conservation or new playground for environmental NGOs?", Communication présentée à la conférence finale du programme CORUS- DDP, 22 octobre 2007 à Durban North, actes sous presse.

Guyot S., Mniki L., 2008, « Les parcs nationaux sud-africains, entre frontières raciales et frontière environnementale : les nouveaux enjeux de la conservation de la nature sur la Wild Coast », in Héritier S. et Laslaz L., Les parcs nationaux dans le monde, Paris, Ellipses, sous presse.

Guyot S., Rey B., 2003, “ De la conservation du patrimoine naturel à une tentative de développement communautaire? Le cas du Maputaland, Afrique du Sud "in Patrimoines et développement dans les pays tropicaux (actes des Journées de Géographie Tropicale de la Rochelle, septembre 2001), P. Cosaert et F. Bart (dirs.), Pessac : Dymset/Cret (Espaces tropicaux no 1), p. 235-246.

Mniki L, 2007, "Subsistence fishermen on the Wild Coast", Communication présentée à la conference finale du programme CORUS- DDP, 22 octobre 2007 à Durban North.

Nicholas C., 1997, «Richards Bay, a rough diamond ». Kwazulu Natal Brieffing, n 9, Houghton, The Helen Suzmann Foundation

Robbins P., 2004, Political Ecology, Malden, Critical Introductions to Geography, Blackwell Publishing, $242 \mathrm{p}$.

Weulersse J., 1931, Noirs et Blancs, Paris, Editions du CTHS (réédition de 1993)

\section{NOTES}

1. Définition du littoral reformulée, d'après Hypergéo, accédé le 20/02/2008, http:// www.hypergeo.eu/spip.php?article145

2. Pour reprendre, en s'en distanciant très fortement, le vocabulaire colonialiste et d'apartheid, toujours d'actualité dans le pays. 
3. Définition de «front pionnier », Hypergéo, accédé le 20/02/2008, http://www.hypergeo.eu/ article.php3?id_article=357

4. De manière plus prosaïque, bien sur, l'espace maritime littoral est lui-même contrôlé, borné et obéit à différents types de régulations.

5. Les petits pélagiques vivent dans des eaux entre 13 et $19^{\circ}$. Ils ne migrent donc pas vers les eaux chaudes du Mozambique mais vers une zone de «bloom» planctonique induite par des remontées d'eau froide le long de la côte jusqu'au niveau de Watterbluff, proche de Durban (voir Ligen et al 2006). Les prédateurs suivent les petits pélagiques, ce qui forme le sardine run. Ces remontées d'eau froides le long de la côte se voient parfaitement par télédétection satellitaire (SST des satellites NOAA).

6. Publications sur le littoral du KwaZulu-Natal (voir Guyot, 2002, 2003, 2004) et travail en collaboration avec Julien Dellier et Lizile Mniki sur la Wild Coast, dans la province de l'Eastern Cape (voir Guyot, Mniki \& Dellier, 2007 et Guyot \& Mniki, 2008).

7. Littéralement "paysans", descendants de Huguenots français vignerons, de Hollandais et d'Allemands. De nombreux métissages auront lieu avec des populations Khoisan et des esclaves malais et indonésiens, ce qui formera la catégorie raciale " coloured " pendant l'apartheid.

8. VOC : Vereenigde Oostindische Compagnie

9. Ce sont des idées libérales à la fois humanistes (abolition de l'esclavage) et économiques (capitalisme, système de la mine, industrialisation et première division internationale du travail...conduisant à d'autres formes d'exploitation...par le travail).

10. Port Alfred, Port St Johns, Port Edward et Port Shepstone, comme leur nom ne l'indique pas, ne sont pas des ports. Il y eut à Port St Johns et Port Shepstone quelques tentatives portuaires par le biais de wharfs mais elles ne résistèrent pas longtemps à l'ensablement ou aux tempêtes. Port Alfred, en revanche, peut être maintenant qualifié de port de plaisance depuis que Justin Steyn y a développé dans les années 1980 une marina en s'inspirant du modèle français de Port Grimaud. L'entrée maritime dans la marina reste toutefois très aléatoire en fonction des vents, parfois violents, qui soufflent sur la Sunshine Coast (communication personnelle de J. Steyn, 25 novembre 2007, Port Alfred).

11. Lire à ce propos la description des petits blancs Afrikaners faite par J. Weulersse, 1931, dans « Noirs et Blancs », Paris, Editions du CTHS (réédition de 1993) dans le train côtier entre George et Knysna (pp 226-227).

12. Contrepoint humoristique à la notion de "terre promise » biblique recherchée par les Huguenots en Afrique du Sud à leur arrivée, et confortée par le départ du grand Trek.

13. Le goût des Afrikaners pour les caravanes transcende largement l'épisode du grand Trek.

14. Au même moment sera créé son " pendant atlantique »: Saldanha Baai sur la West Coast au nord du Cap.

15. Au moment de l'inauguration du parc national Kruger.

16. Un autre projet de création portuaire est initié directement par Pretoria à la fin des années 1970. Il s'agit de construire un port pour le petit royaume du Swaziland, Etat intérieur enclavé. Il serait localisé au niveau de la lagune de Kosi Bay. En effet, le Swaziland soutient le régime de l'apartheid. Ce serait donc une forme de récompense territoriale que de lui ménager un accès à la mer. Pourtant, le leader Zulu, Buthelezi, refuse sous le prétexte d'une violation territoriale des terres royales zulu (autrefois tongas...) et aussi en raison des impacts environnementaux potentiellement très importants. Effectivement, le projet de réserve naturelle pour Kosi Bay est déjà à l'étude.

17. Entreprise connue aujourd'hui sous le nom de "GTM construction», groupe Vinci. Elle a participé à la construction du barrage de Castillon sur Verdon entre 1928 et 1948. De nombreuses entreprises françaises ont utilisé le système des Bantoustans pour investir en Afrique du Sud. Ainsi la majorité des stations services du Transkei sont des stations Total. 
18. La toponymie suggère d'elle-même l'appropriation coloniale européenne du littoral « indien » d'Afrique du Sud.

19. Parti nationaliste zulu à base rurale et à fond idéologique traditionnel, opposé à l'ANC fer de lance de l'opposition anti-apartheid.

20. Election de Mandela à la présidence de la nouvelle démocratie multiraciale

21. On peut ajouter à ces chiffres une bande de protection littorale d'un kilomètre de large et d'environ $280 \mathrm{~km}$ de long édictée du temps du bantoustan du Transkei par B. Holomisa et qui contraint encore plus (en théorie) les aménagements littoraux.

22. Cette proportion se retrouve de manière équivalente sur tout le littoral « indien » d'Afrique du Sud.

23. Il est très difficile d'évaluer la population du littoral « indien » d'Afrique du Sud. Le résultat le plus rapproché pourrait être calculé en faisant la somme démographique de tous les arrondissements littoraux de tous les municipalités ayant une zone côtière dans leur territoire. Il faut rappeler que les nouvelles municipalités sud-africaines sont de grande taille... C'est donc un travail assez fastidieux.

24. Les ports industriels de Port Elizabeth, Est London et Durban sont maintenant partiellement contrôlés par des élus et des hommes d'affaires noirs. Ce nouveau contrôle participe au développement de périmètres métropolitains cohérents.

25. Initiatives de développement spatial

26. Statistiques électorales : www.elections.org.za (Independant Electoral Commission)

27. Les sérieux problèmes de production électriques rencontrés par l'Afrique du Sud depuis 2 ans ne sont pas du meilleur effet pour attirer de telles industries.

28. Jacob Zuma, homme politique de l'ANC de langue zulu, a été mis en examen pour corruption en décembre 2007 dans le cadre d'un marché d'armements impliquant le groupe français THALES.

29. La pression foncière sur les terres littorales, comme intérieures, des ex-bantoustans est importante. Toutefois la propriété et l'usage de ces terres est théoriquement exclu du marché foncier globalisé pour être réservé aux communautés locales.

30. A Port St Johns, $2^{\text {nd }}$ Beach, en novembre 2007, l'alcool sur la plage n'est pas contrôlé : il en résulte un grand laisser-aller au grand dam des touristes internationaux venus goûter la tranquillité des lieux!

31. Propos de Liz Wood en 2001 à propos de la plage municipale de Richards Bay: "It should not be a public beach. If we made that over to the Parks, they could upgrade it like they did in Mtunzini, they have a beautiful place there, very, very nice and many of the other resorts up and down the coast are controlled by Parks. They have got the background, they have got the knowledge, they've got the expertise, they have got the know how. I think they do a brilliant job, a very good job. We just haven't got the know how and we haven't got the big bucks."Ces propos renvoient très bien à la tendance actuelle d'occupation des plages en Afrique du Sud (voir Guyot, 2006).

32. Forme sud-africaine du pick-up ou de la camionnette.

33. Voir l'article très récent du Mail \& Guardian en ligne du 22/02/2008 : « Big stink over R9bn Coega prawn farm ». www.mg.co.za

34. Le littoral du Transkei est resté pendant longtemps dans les représentations de beaucoup de sud-africains blancs comme un espace sacrifié à l'indigène et totalement infréquentable en raison des problèmes d'insécurité. Ce genre de représentations tenaces facilite grandement le travail des conservationnistes qui souhaitent protéger la nature pour l'usage d'une petite minorité sous fond de discours intégratif envers les "communautés locales", tout comme les projets miniers qui, au final, profitent aussi à peu de monde. 


\section{RÉSUMÉS}

Le littoral « indien » en Afrique du Sud représente un véritable « front pionnier côtier », dont la conquête, l'appropriation territoriale et la valorisation économique (portuaire, industrielle, touristique, environnementale, agricole) sont stratégiques pour différents groupes de populations (Anglophones, Afrikaner, Zulus, Xhosas...), à différentes périodes temporelles (précolonial, colonial, apartheid et postapartheid). Ces différents modes de valorisation ainsi que les différents groupes en jeu entrent régulièrement en conflit tout en se recomposant au fil des temps. Dans cet article, à l'aide d'exemples croisant temporalités, acteurs et modes de valorisation littorale, nous caractérisons les différentes phases de cette conquête littorale, toujours d'actualité depuis la fin de l'apartheid en raison de la connexion de l'ensemble des zones côtières aux processus de globalisation. La conquête de cette ultime «frontière australe de l'Afrique » par des forces essentiellement exogènes présente un certain nombre de limites, comme la difficile intégration des populations locales, la privatisation de l'espace côtier et une durabilité environnementale mal maîtrisée.

The "Indian" south African coastline can be understood as a frontier. Its conquest is strategic for different groups (English-speaking, Afrikaners, Zulus, Xhosas etc.) and implies specific economic valorisations (seaports, industries, tourism, nature conservation, agriculture) grounded in time (pre-colonial, colonial, apartheid and post-apartheid) and space (different modes of territorial appropriation). Opposed types of coastal valorisation and various stakeholders open the way to hard conflicts. Different phases of this seafront conquest, with particular reference to the current post-apartheid connection to globalisation dynamics, are enlightened by examples linking history, stakeholders and various coastal development options. The "neo-colonisation process" of this southern frontier of the African continent by outside forces implies problematic stakes like poor integration of local people, privatisation of the coastal zone and contradictory environmental management.

\section{INDEX}

Keywords : coastal resort, conquest, frontier, Indian ocean coastline, industrial seaport, mode of valorisation, national park, South Africa, stakeholder, temporality

Mots-clés : acteur, Afrique du Sud, conquête, front pionnier, littoral « indien », mode de valorisation, parc national, port industriel, station balnéaire, temporalité

\section{AUTEURS}

\section{SYLVAIN GUYOT}

Sylvain Guyot (sylvain.guyot@unilim.fr) est maître de conférences en géographie à l'université de Limoges et est membre de l'UMR 6042-CNRS GEOLAB. Il travaille sur l'Afrique du Sud depuis 1997, sur le littoral du KwaZulu-Natal (géographie politique de l'environnement), puis sur les changements de noms de lieux. Son programme de recherche actuel (C2R, Contrat Renforcé de Recherche) concerne la Wild Coast, en collaboration avec l'Université de Fort Hare avec un travail plus théorique sur la notion de front écologique au croisement des dynamiques territoriales, foncières et de mise en réseau. Depuis 2005, il investit de nouveaux terrains dans les Andes (Chili, Argentine, Bolivie) sur des problématiques similaires. Il a récemment publié : 
- Guyot S., Mniki L., 2008, Les parcs nationaux sud-africains, entre frontières raciales et « frontière environnementale " : Les nouveaux enjeux de la conservation de la nature sur la Wild Coast., in : Héritier S. et Laslaz L. Les parcs nationaux dans le monde. Ellipses, Paris, à paraître en octobre 2008.

- Giraut F., Guyot S., Houssay M., 2008, Enjeux de mots: les changements toponymiques sudafricains, Espace Géographique 2008-2, 131-150.

- Giraut F., Houssay M. Guyot S. (avec la collaboration de), 2008, Au nom des territoires! Enjeux géographiques de la toponymie (introduction au dossier de l'EG sur la toponymie), Espace Géographique 2008-2, 97-105.

\section{JULIEN DELLIER}

Julien Dellier (julien.dellier@unilim.fr) est post-doctorant C2R et est hébergé par l'UMR 6042CNRS GEOLAB. 